\title{
Where do I Go: Sistema de Recomendações Turísticas
}

\author{
${ }^{1}$ Mauricio P. Freitas, ${ }^{1}$ Anita Maria da Rocha Fernandes, ${ }^{2}$ Mônica Rodrigues da \\ Silva \\ ${ }^{1}$ Curso Ciência da Computação - Universidade do Vale do Itajaí (UNIVALI) Campus \\ Kobrasol - São José - SC - Brasil \\ ${ }^{2}$ MBA em Big Data - FATEC - SENAI - Cuaibá - MT \\ mauriciopasetto@edu.univali.br, anita.fernandes@univali.br; \\ monikinha.r@gmail.com
}

\begin{abstract}
This paper describes the development of Where do I Go, a Mobile Tourist Recommendations System that uses recommendation of interest points according to user profile, temporal and semantic constraints, using Case Based Reasoning (CBR). The application aims to make recommendations to tourists during the experimentation of the city, which are in accordance with their tourism preferences and the context at the time of recommendation. CBR stores knowledge around a particular domain in case format, where each case has a problem part and another solution. CBR is premised on the fact that similar problems have similar solutions, where the basis for solving new problems is previously solved problems.
\end{abstract}

Resumo. Este artigo descreve o desenvolvimento do Where do I Go, um Sistema de Recomendações Turísticas para dispositivos móveis, que utiliza recomendação de pontos de interesse de acordo com o perfil do usuário, restrições temporais e semânticas utilizando o Raciocínio Baseado em Casos (RBC). A aplicação tem como objetivo, efetuar recomendações aos turistas durante a experimentação da cidade, que estejam de acordo com suas preferências de turismo e do contexto no momento da recomendação. $O R B C$ armazena conhecimento a cerda de um determinado domínio no formato de casos, onde cada caso possui uma parte problema e outra solução. O RBC tem como premissa o fato de que problemas similares possuem soluções similares, onde a base para resolução de novos problemas são problemas resolvidos anteriormente.

\section{Introdução}

Dentro do conceito de Smart Cities aplicado ao setor do turismo, mas com aplicações específicas com foco no destino turístico e na indústria do turismo, está o conceito Smart Tourism, este tem como objetivo melhor da qualidade de vida do morador e do visitante e de aumentar a qualidade da experiência turística. Uma ferramenta importante para o Smart Tourism são os Sistemas de Recomendação de Turismo (SRT), como instrumento de apoio a decisões inteligentes e de personalização de conteúdo [Roopesh e Tulasi 2018].

O Where do I Go é um sistema personalizado de recomendação de pontos de interesse (PI), com o objetivo de efetuar recomendações aos turistas durante a 
experimentação da cidade, que estejam de acordo com suas preferências de turismo e do contexto no momento da recomendação. Para efetuar as recomendações o sistema apresentado neste artigo utiliza o Raciocínio Baseado em Casos (RBC), que é "Inferência ou técnica de resolução de problemas baseado na similaridade entre o problema atual e os previamente resolvidos que são armazenados em um repositório" [Dubois e Prade 2018, p.1]. O RBC foi selecionado por ser uma técnica madura usada para a resolução de grande espectro de problemas, que vão desde identificar causas de conflitos no casamento [Ichwani e Suprato, 2019] até a detecção de emergências em usinas nucleares [Raskob; Möhrle; Bai, 2016]. O problema aqui é efetuar recomendações de PI de acordo com o perfil do usuário.

Disponibilizar os serviços para clientes através de uma aplicãção móvel deve-se a grande utilização do dispositivo pela sociedade atual [FGV, 2019], e pelo fato de que o sistema efetuará recomendações utilizando o serviço de localização disponíveis nos telefones celulares. Isto permitirá aos usuários solicitarem recomendações durante a experimentação da cidade. Por sua vez, o cliente móvel irá consumir os recursos disponibilizados via Web Service RestFull. o que permite a implementação de clientes para diferentes plataformas como Web, Android e IOS.

\section{Visão geral do sistema}

Em relação a arquitetura do sistema, o back-end será implementado utilizando Web Service RESTFull, disponibilizando as funcionalidades através de serviços para o frontend, este último a ser disponibilizado para dispositivos móveis Android. Nos serviços estão incluídos, o gerenciamento do perfil do usuário, a autenticação de usuários e as funcionalidades de recomendação de pontos de interesse. Foram selecionadas para a implementação das camadas: o React para o front-end, o Java para a implementação do back-end e para a persistência o MySql.

Em relação ao Perfil do Usuário o sistema utiliza o modelo de vetor com avaliações numéricas, onde o usuário define o interesse para as categorias de turismo pré-definidas, sendo elas: cultural, ecológico, gastronômico, compras e religioso.

Em relação ao método de filtragem, o sistema utiliza o conhecimento à cerca dos PI no formato de casos a partir da API Google Places e da Base de Casos.

Em relação as funcionalidades a serem implementadas, tem-se: a recomendação de PI com restrições temporais (disponibilidade de visitação no período selecionado), semânticas (localização e investimento necessário), a recomendação de rota (utilizando API Google Maps) e o gerenciamento da lista Interesse na Visitação do usuário.

Em relação ao RBC, o sistema utiliza o ciclo de 4 fases, esta é a parte mais crítica do sistema, pois é responsável por efetuar as recomendações. A fase de recuperação, calcula a similaridade entre o problema atual (perfil do usuário e restrições) e os casos existentes (casos montados em memória a partir da API Google Places e a Base de Casos a ser implementada), os casos retornados de diferentes bases são mesclados para compor a recomendação apresentada ao usuário.

As fases de reutilização, revisão e retenção de casos utilizam a seguinte abordagem: a reutilização é feita de forma inalterada, ou seja, não haverá modificações na parte da solução do problema; a revisão ocorre através do feedback do usuário em relação aos itens recomendados, isto ocorre de forma implícita quando o usuário 
adiciona o item recomendado à lista de Interesse na Visitação; a retenção se inicia quando o caso passa pela fase de revisão, desta forma o caso é incorporado à base de casos criando um novo caso vinculando o problema de pesquisa (perfil do usuário e restrições) e a solução proposta (ponto de interesse).

\section{Conclusões}

Em relação ao Smart Tourism, o sistema contribui para aumentar a qualidade da experiência de turismo, na medida em que fornece recomendações personalizadas de acordo com o perfil e contexto do turista, fazendo com que as experiências sejam mais ricas e significativas.

Do ponto de vista econômico, contribui na medida que novos atrativos são descobertos e experimentados pelo turista, fomentando investimento por parte do usuário.

Em relação ao $\mathrm{RBC}$ aplicado no problema de pesquisa deste trabalho, altos valores de similaridade (próximos de um) indicam um grau maior de personalização (recomendações mais assertivas) e o contrário para valores baixos de similaridade, pois, a parte do problema representa o perfil do usuário e características dos pontos de interesse.

Outra questão que deve ser levada em conta é o tamanho e a abrangência da base de casos, que contribui diretamente na qualidade das recomendações. Um detalhe importante em relação ao ciclo $\mathrm{RBC}$, é o fato de que as fases podem ser adaptadas e utilizarem diferentes abordagens dependendo do tipo do problema que se busca resolver com o RBC. Neste trabalho a fase de recuperação de casos foi adaptada para buscar casos na base da API Google Places, a qual pode ser estendida para utilizar outras bases adicionando novos métodos de recuperação e ajustando o método de mesclagem dos resultados.

As próximas etapas do trabalho se referem a implementação do sistema. E consiste na criação da base de dados, o desenvolvimento do back-end da aplicação, o desenvolvimento do front-end, o teste da aplicação e a aplicação e avaliação do experimento.

\section{Referências}

Dubois, D.; Prade, H. (2008) Handbook of Research on Fuzzy Information Processing in Databases. IGI Global.

FGV EAESP. "Pesquisa Anual do uso de TI". 2019.

Ichwani, A.; Suprapto, S. "Cased Based Reasoning to Identify Cause Conflicts in Marriage". In: Indonesian Journal of Computing and Cybernetics Systems, v. 13, n. 1, p. 1-10, January 2019.

Roopesh, L.R., Tulasi, B. "A Survey of Travel Recommender System”. In: International Journal of Computer Sciences and Engeneering Open Access, Bengaluru, v. 6, n. 2347-2693, September 2018.

Raskob, W.; Möhrle, S.; Bai, S. "Knowledge database and case-based reasoning". In: Radioprotection, v. 51, p. 185-186, December 2016. 